\title{
A NEW APPROACH TO STUDY HYPERBOLIC-PARABOLIC COUPLED SYSTEMS
}

\author{
YA-GUANG WANG \\ Department of Mathematics \\ Shanghai Jiao Tong University \\ 200030 Shanghai, P. R. China \\ E-mail: ygwang@sjtu.edu.cn
}

1. Introduction. In many physical problems, like in thermoelasticity, viscous fluid etc., people often meet hyperbolic-parabolic coupled systems. There is a rich literature devoted to the existence, regularity and long time asymptotics of solutions to initial (-boundary) value problems for linear and nonlinear hyperbolic-parabolic coupled systems, mostly relying on the framework of semigroup theory or of abstract evolutional equations, cf. $[11,15,21]$ and references therein. To describe properties of solutions precisely, it would be very interesting and useful if one could decouple the hyperbolic and parabolic operators in the coupled systems. In this note, we shall survey our recent works in developing a general procedure to weakly decouple the hyperbolic-parabolic systems as well as the applications. The decoupling argument is inspired by Taylor's work [18], in which an idea was introduced to decouple the different characteristic fields for studying the reflection of singularities in hyperbolic problems. In Section 3, by using the decoupling argument, several interesting applications will be given ranging from the propagation of singularities either in nonlinear thermoelasticity or in viscous fluids, to find that there is a cone of dependence for the propagation of singularities in nonlinear coupled systems, which shows that the hyperbolic operators in coupled systems play a dominant role in determining the regularity of solutions.

2. A general idea for decoupling hyperbolic-parabolic coupled systems. For any given $\Omega \subseteq \mathbb{R}^{n}$, denote by $\Psi^{k}(\Omega)$ the set of pseudodifferential operators of order $k$ with symbols in $S^{k}\left(\Omega \times \mathbb{R}_{\xi}^{n}\right)([3,10])$. Consider the following linear hyperbolic-parabolic

This research was partially supported by the NSFC and the Educational Ministry of China. 2000 Mathematics Subject Classification: Primary 35M10, 35A21; Secondary 35Q30, 80A17. The paper is in final form and no version of it will be published elsewhere. 
coupled system in $\mathbb{R}_{t} \times \mathbb{R}_{x}^{n}$ :

$$
\left\{\begin{array}{r}
\partial_{t} u+\sum_{j=1}^{n} A_{j}(t, x) \partial_{x_{j}} u+\sum_{j=1}^{n} B_{j}(t, x) \partial_{x_{j}} v+A_{0}(t, x) u+B_{0}(t, x) v=f(t, x) \\
\partial_{t} v-\sum_{j, k=1}^{n} a_{j k}(t, x) \partial_{x_{j} x_{k}}^{2} v+\sum_{j=1}^{n} C_{j}(t, x) \partial_{x_{j}} u+\sum_{j=1}^{n} d_{j}(t, x) \partial_{x_{j}} v \\
+C_{0}(t, x) u+d_{0}(t, x) v=g(t, x)
\end{array}\right.
$$

where all coefficients are supposed to be smooth for simplicity, $u=\left(u_{1}, \ldots, u_{N}\right)^{T}(t, x)$, $v(t, x)$ is scalar, $\left\{A_{j}(t, x)\right\}_{j=0}^{n}$ are $N \times N$ matrices, $\left\{B_{j}(t, x)\right\}_{j=0}^{n}$ are $N \times 1$ vectors, $\left\{C_{j}(t, x)\right\}_{j=0}^{n}$ are $1 \times N$ vectors, $\left\{d_{j}(t, x)\right\}_{j=0}^{n}$ are scalar, and $\left(a_{j k}(t, x)\right)_{n \times n}$ is positively definite, which means there is $a_{0}>0$ such that

$$
\sum_{j, k=1}^{n} a_{j k}(t, x) \xi_{j} \xi_{k} \geq a_{0}|\xi|^{2}
$$

for any $\xi=\left(\xi_{1}, \ldots, \xi_{n}\right) \in \mathbb{R}^{n}$. We are going to seek for a transformation of $(u, v)$ in order to decouple the system $(2.1)$. Let $\Lambda_{a}=\left(1-\sum_{j, k=1}^{n} a_{j k} \partial_{x_{j} x_{k}}^{2}\right)^{\frac{1}{2}}$, then from (2.1) we know that $U=(u, v)^{T}$ satisfies the system

$$
\partial_{t} U+H_{2}\left(t, x, D_{x}\right) U+H_{1}\left(t, x, D_{x}\right) U+H_{0}\left(t, x, D_{x}\right) U=F(t, x)
$$

where $F=(f, g)^{t}, H_{2}\left(t, x, D_{x}\right)=\operatorname{diag}\left[\mathbf{0}_{N \times N}, \Lambda_{a}^{2}\right] \in \Psi^{2}\left(\mathbb{R}^{n}\right)$ and $H_{1} \in \Psi^{1}\left(\mathbb{R}^{n}\right)$, $H_{0} \in \Psi^{0}\left(\mathbb{R}^{n}\right)$ are given obviously by $(2.1)$. As in [18], let $U^{(1)}=\left(I+K_{1}\right) U$ where $K_{1}\left(t, x, D_{x}\right) \in \Psi^{-1}\left(\mathbb{R}^{n}\right)$ will be determined later, then from (2.2) we have

$$
\begin{aligned}
\partial_{t} U^{(1)}+H_{2} U^{(1)}+ & H_{1} U^{(1)}+\left[K_{1}, H_{2}\right] U \\
& +\left(\left[K_{1}, H_{1}\right]-\partial_{t} K_{1}+\left(I+K_{1}\right) H_{0}\right) U=\left(I+K_{1}\right) F .
\end{aligned}
$$

Temporarily, we assume that $I+K_{1}$ is invertible, which will be realized by slightly modifying $K_{1}$ later. Then, by using $\left(I+K_{1}\right)^{-1}=I-K_{1}\left(I+K_{1}\right)^{-1}$, the system (2.3) can be rewritten as the following one for $U^{(1)}$,

$$
\partial_{t} U^{(1)}+H_{2} U^{(1)}+\left(\left[K_{1}, H_{2}\right]+H_{1}\right) U^{(1)}+H_{0}^{(1)} U^{(1)}=F^{(1)},
$$

where $F^{(1)}=\left(I+K_{1}\right) F$, and

$$
H_{0}^{(1)}=\left(\left[K_{1}, H_{1}\right]+\left(I+K_{1}\right) H_{0}-\partial_{t} K_{1}-\left[K_{1}, H_{2}\right] K_{1}\right)\left(I+K_{1}\right)^{-1} \in \Psi^{0}\left(\mathbb{R}^{n}\right) .
$$

Noting that the eigenvalues of the symbol of $H_{2}\left(t, x, D_{x}\right)$ are zero and $\langle\xi\rangle_{a}^{2}:=1+$ $\sum_{j, k=1}^{n} a_{j k} \xi_{j} \xi_{k}$ with the multiplicity of zero being $N$, as in [18], if we define $K_{1}(t, x, \xi)$ as

$$
K_{1}(t, x, \xi)=\left(\begin{array}{cc}
\mathbf{0}_{N \times N} & K_{12} \\
K_{21} & 0
\end{array}\right)
$$

with $K_{12}$ of dimension $N \times 1$, and set the matrix

$$
K_{1}(t, x, \xi) H_{2}(t, x, \xi)-H_{2}(t, x, \xi) K_{1}(t, x, \xi)+H_{1}(t, x, \xi)
$$

to be the block form

$$
\left(\begin{array}{cc}
J_{1} & 0 \\
0 & J_{2}
\end{array}\right)
$$


with $J_{1}$ of dimension $N \times N$, then we can easily obtain

$$
K_{1}(t, x, \xi)=i\langle\xi\rangle_{a}^{-2}\left(\begin{array}{cc}
\mathbf{0}_{N \times N} & -\sum_{j=1}^{n} B_{j}(t, x) \xi_{j} \\
\sum_{j=1}^{n} C_{j}(t, x) \xi_{j} & 0
\end{array}\right) \in S^{-1}\left(\mathbb{R}^{n}\right)
$$

and

$$
J_{1}=i \sum_{j=1}^{n} A_{j}(t, x) \xi_{j}, \quad J_{2}=i \sum_{j=1}^{n} d_{j}(t, x) \xi_{j}
$$

Obviously, there is $M>0$ such that when $|\xi| \geq M, I+K_{1}(t, x, \xi)$ is invertible. Hence, we can find a smooth symbol

$$
\tilde{K}_{1}(t, x, \xi)= \begin{cases}K_{1}(t, x, \xi), & \text { when }|\xi| \geq M \\ 0, & \text { when }|\xi| \ll 1,\end{cases}
$$

such that $I+\tilde{K}_{1}(t, x, \xi)$ is invertible for any $\xi \in \mathbb{R}^{n}$, and

$$
U^{(1)}=\left(I+\tilde{K}_{1}\left(t, x, D_{x}\right)\right) U
$$

satisfies

$$
\partial_{t} U^{(1)}+H_{2}\left(t, x, D_{x}\right) U^{(1)}+H_{1}^{(1)}\left(t, x, D_{x}\right) U^{(1)}+H_{0}^{(1)}\left(t, x, D_{x}\right) U^{(1)}=F^{(1)},
$$

where $H_{1}^{(1)}\left(t, x, D_{x}\right)=\operatorname{diag}\left[\sum_{j=1}^{n} A_{j}(t, x) \partial_{x_{j}}, \sum_{j=1}^{n} d_{j}(t, x) \partial_{x_{j}}\right]$, and $H_{0}^{(1)}\left(t, x, D_{x}\right)$ belongs to $\Psi^{0}\left(\mathbb{R}^{n}\right)$. Next, if we define $U^{(2)}=\left(I+K_{2}\left(t, x, D_{x}\right)\right) U^{(1)}$ with $K_{2} \in \Psi^{-2}\left(\mathbb{R}^{n}\right)$, then by the same procedure as above we can find $K_{2}$ such that $U^{(2)}$ satisfies a system with the same second and first order terms as in (2.5), its zero-th order term is decoupled, and $I+K_{2}(t, x, \xi)$ is invertible. In this way, we can decouple further terms by setting $U^{(j)}=\left(I+K_{j}\left(t, x, D_{x}\right)\right) U^{(j-1)}$ with $K_{j} \in \Psi^{-j}\left(\mathbb{R}^{n}\right)$ for any $j \geq 3$. For any fixed integer $m \in \mathbb{N}$, if we define $K\left(t, x, D_{x}\right) \in \Psi^{-1}\left(\mathbb{R}^{n}\right)$ by

$$
I+K=\left(I+K_{m+1}\right) \cdots\left(I+K_{2}\right)\left(I+\tilde{K}_{1}\right)
$$

then $V=\left(I+K\left(t, x, D_{x}\right)\right) U$ satisfies the following decoupled system modulo $\Psi^{-m}$ :

$$
\partial_{t} V+H_{2}\left(t, x, D_{x}\right) V+H_{1}^{(1)}\left(t, x, D_{x}\right) V+H_{0}\left(t, x, D_{x}\right) V+R_{m}\left(t, x, D_{x}\right) V=\mathcal{F},
$$

where $R_{m} \in \Psi^{-m}$, and $H_{0}\left(t, x, D_{x}\right)=\operatorname{diag}\left[H_{0}^{(1)}, H_{0}^{(2)}\right] \in \Psi^{0}\left(\mathbb{R}^{n}\right)$ with $H_{0}^{(1)}$ being $N \times N$ and $H_{0}^{(2)}$ scalar.

REMARK 2.1.

(1) Obviously, the above procedure also works for the case that the second equation in (2.1) is replaced by a parabolic system.

(2) If (2.1) is a quasilinear coupled system, we can decouple it by using paradifferential operators (see Section 3.3).

\section{Applications}

3.1. Finite speeds for the propagation of singularities in coupled systems. As the first application of our decoupling idea, we shall show that hyperbolic-parabolic coupled systems still have finite speeds for the propagation of singularities as for the case of pure hyperbolic equations. 
ThEOREM 3.1. Consider the Cauchy problem for the semilinear version of hyperbolicparabolic coupled system (2.1):

$$
\left\{\begin{array}{l}
\partial_{t} u+\sum_{j=1}^{n} A_{j}(t, x) \partial_{x_{j}} u+\sum_{j=1}^{n} B_{j}(t, x) \partial_{x_{j}} v=f(u, v) \\
\partial_{t} v-\sum_{j, k=1}^{n} a_{j k}(t, x) \partial_{x_{j} x_{k}}^{2} v+\sum_{j=1}^{n} C_{j}(t, x) \partial_{x_{j}} u+\sum_{j=1}^{n} d_{j}(t, x) \partial_{x_{j}} v=g(u, v) \\
t=0: u=u_{0}(x), v=v_{0}(x)
\end{array}\right.
$$

with all notation being the same as in (2.1). For any open $\omega \subseteq \mathbb{R}^{n}$, let $\Omega \subseteq[0,+\infty) \times \mathbb{R}^{n}$ be the determinacy domain of $\omega$ with respect to the hyperbolic operator

$$
\partial_{t}+\sum_{j=1}^{n} A_{j}(t, x) \partial_{x_{j}} .
$$

If the initial data satisfy

$$
u_{0} \in H^{s}\left(\mathbb{R}^{n}\right) \cap C^{\infty}(\omega), \quad v_{0} \in H^{s+1}\left(\mathbb{R}^{n}\right) \cap C^{\infty}(\omega)
$$

for a fixed $s>n / 2$, then there is $T>0$ such that

$$
(u, v) \in C^{\infty}(\Omega \cap\{0 \leq t<T\}) .
$$

Proof. By employing the decoupling technique for the problem (3.1), it follows that $V(t, x)=\left(V_{1}, V_{2}\right)^{T}=\left(I+K\left(t, x, D_{x}\right)\right)(u, v)^{T}$ satisfies the Cauchy problem

$$
\left\{\begin{array}{l}
\partial_{t} V_{1}+\sum_{j=1}^{n} A_{j}(t, x) \partial_{x_{j}} V_{1}=\mathcal{F}_{1}\left(V_{1}, V_{2}\right) \\
\partial_{t} V_{2}-\sum_{j, k=1}^{n} a_{j k}(t, x) \partial_{x_{j} x_{k}}^{2} V_{2}+\sum_{j=1}^{n} d_{j}(t, x) \partial_{x_{j}} V_{2}=\mathcal{F}_{2}\left(V_{1}, V_{2}\right) \\
t=0: V_{1}=V_{1}^{(0)}(x), V_{2}=V_{2}^{(0)}(x)
\end{array}\right.
$$

with $\left(V_{1}^{(0)}(x), V_{2}^{(0)}(x)\right)^{T}=\left(I+K\left(0, x, D_{x}\right)\right)\left(u_{0}, v_{0}\right)^{T}$. Under assumption (3.2), we have

$$
V_{1}^{(0)} \in H^{s}\left(\mathbb{R}^{n}\right) \cap C^{\infty}(\omega), \quad V_{2}^{(0)} \in H^{s+1}\left(\mathbb{R}^{n}\right) \cap C^{\infty}(\omega) .
$$

Applying the classical theory of hyperbolic and parabolic equations to (3.4), we obtain the following estimate for the norm of $\left(V_{1}, V_{2}\right)$ in $C\left([0, T], H^{s}\left(\mathbb{R}^{n}\right)\right) \times C\left([0, T], H^{s+1}\left(\mathbb{R}^{n}\right)\right)$ :

$$
\begin{aligned}
& \left\|V_{1}(t, \cdot)\right\|_{H^{s}}^{2}+\left\|V_{2}(t, \cdot)\right\|_{H^{s+1}}^{2} \\
& \quad \leq C_{0}\left(\left\|V_{1}^{(0)}\right\|_{H^{s}}^{2}+\left\|V_{2}^{(0)}\right\|_{H^{s+1}}^{2}+\int_{0}^{t}\left(\left\|\mathcal{F}_{1}(V)\left(t_{1}, \cdot\right)\right\|_{H^{s}}^{2}+\left\|\mathcal{F}_{2}(V)\left(t_{1}, \cdot\right)\right\|_{H^{s}}^{2}\right) d t_{1}\right)
\end{aligned}
$$

with a constant $C_{0}>0$, which easily implies the existence and uniqueness of the solution $V=\left(V_{1}, V_{2}\right)^{T} \in C\left([0, T], H^{s}\left(\mathbb{R}^{n}\right)\right) \times C\left([0, T], H^{s+1}\left(\mathbb{R}^{n}\right)\right)$ to (3.4) for certain $T>0$ by using a classical argument. From the regularity of parabolic equations, we also have

$$
V_{2} \in L^{2}\left(0, T ; H^{s+2}\left(\mathbb{R}^{n}\right)\right) \cap H^{1}\left(0, T ; H^{s}\left(\mathbb{R}^{n}\right)\right) .
$$

Using (3.6) and applying the theory of hyperbolic equations (cf. [1,4]) to problem (3.4) for $V_{1}$, we obtain

$$
V_{1} \in C\left([0, T], H_{\mathrm{loc}}^{s+2}\left(\omega_{t}\right)\right) \cap C^{1}\left([0, T], H_{\mathrm{loc}}^{s+1}\left(\omega_{t}\right)\right)
$$


where $\omega_{t}=\Omega \cap\{t=$ const. $\}$. Denoting by $C_{0}^{\infty}\left(\bar{\Omega}_{T}\right)$ the set of smooth functions infinitely order vanishing on $\partial \Omega_{T} \backslash\{t=0, T\}$, and letting $\chi \in C_{0}^{\infty}\left(\bar{\Omega}_{T}\right)$, from (3.4) we know that $\chi V_{2}$ satisfies

$$
\left\{\begin{array}{l}
\left(\partial_{t}-\sum_{j, k=1}^{n} a_{j k}(t, x) \partial_{x_{j} x_{k}}^{2}+\sum_{j=1}^{n} d_{j}(t, x) \partial_{x_{j}}\right)\left(\chi V_{2}\right)=\mathcal{F}_{2, \chi}\left(V_{1}, V_{2}\right) \\
\left(\chi V_{2}\right)(0, x)=\chi(0, x) V_{2}^{(0)}(x)
\end{array}\right.
$$

where $\mathcal{F}_{2, \chi}(V)=\chi \mathcal{F}_{2}(V)+[L, \chi] V_{2}$ belongs to $L^{2}\left(0, T ; H^{s+1}\left(\mathbb{R}^{n}\right)\right)$ by using $(3.6),(3.7)$. Here, $L$ denotes the parabolic operator appearing on the left-hand side of (3.8). Employing the classical theory of parabolic equations for (3.8), we deduce that

$$
\chi V_{2} \in L^{2}\left(0, T ; H^{s+3}\left(\mathbb{R}^{n}\right)\right) \cap H^{1}\left(0, T ; H^{s+1}\left(\mathbb{R}^{n}\right)\right)
$$

which implies

$$
V_{2} \in L^{2}\left(0, T ; H_{\mathrm{loc}}^{s+3}\left(\omega_{t}\right)\right) \cap H^{1}\left(0, T ; H_{\mathrm{loc}}^{s+1}\left(\omega_{t}\right)\right)
$$

from the arbitrariness of $\chi$. Thus, we can continue this process and eventually obtain the conclusion.

REMARK 3.1. It is not difficult to see that the above result holds as well for the case that the nonlinear function $f$ on the right hand side of (3.1) also depends on the first order derivatives of $v$ in space variables.

3.2. Microlocal analysis in semilinear thermoelastic systems. A typical example of $(3.1)$ is the system of thermoelasticity $([11,15])$. Let us study the following semilinear problem of thermoelasticity in three space variables $x=\left(x_{1}, x_{2}, x_{3}\right) \in \mathbb{R}^{3}$ :

$$
\left\{\begin{array}{l}
u_{t t}-(2 \mu+\lambda) \nabla \operatorname{div} u+\mu \operatorname{rot}(\operatorname{rot} u)+\gamma_{1} \nabla \theta=f\left(u, u_{t}, \nabla u, \nabla \theta, \theta\right) \\
\theta_{t}-\beta^{2} \Delta \theta+\gamma_{2} \operatorname{div} u_{t}=g\left(u, u_{t}, \nabla u, \theta\right),
\end{array}\right.
$$

where all coefficients $\left(\mu, \lambda, \gamma_{1}, \gamma_{2}, \beta\right)$ are smooth functions of $(t, x)$ with $\mu, \lambda+\mu$ and $\beta$ being positive, $\nabla u$ ( $\nabla \theta$ resp.) denotes the gradient of $u$ ( $\theta$ resp.) with respect to the space variables $\left(x_{1}, x_{2}, x_{3}\right)$, and nonlinear functions $f$ and $g$ are smooth in their arguments with $f(0)=g(0)=0$. Here $u$ represents the displacement, and $\theta=T_{a}-T_{0}$ is the temperature difference. The first result on the propagation of weak singularities in thermoelasticity was given in $[16,17]$. Here, by employing the decoupling idea of Section 2, we can obtain rather deeper results on the regularity of solutions to (3.10).

\section{Theorem 3.2.}

(1) For a fixed point $\left(x_{0}, \xi_{0}\right) \in T^{*}\left(\mathbb{R}^{3}\right) \backslash\{0\}$, suppose that $\left(u_{0}, \theta_{0}\right) \in H^{s}\left(\mathbb{R}^{3}\right) \cap$ $H_{m l}^{r}\left(x_{0}, \xi_{0}\right), u_{1} \in H^{s-1}\left(\mathbb{R}^{3}\right) \cap H_{m l}^{r-1}\left(x_{0}, \xi_{0}\right)$ for any fixed $\frac{5}{2}<s \leq r<2 s-\frac{5}{2}$. Let $\gamma(t)=\left\{(t, x(t) ; \tau(t), \xi(t)) \in T^{*}\left(\mathbb{R}^{4}\right) \backslash\{0\}: 0 \leq t<T_{0}\right\}$ be a null bicharacteristic of $\partial_{t}^{2}-(2 \mu+\lambda) \Delta$ or $\partial_{t}^{2}-\mu \Delta$ with $x(0)=x_{0}$ and $\xi(0)=\xi_{0}$. Then the problem (3.10) has a unique solution:

$$
\left\{\begin{array}{l}
u \in C\left([0, T], H^{s} \cap H_{m l}^{r}(x(t), \xi(t))\right) \cap C^{1}\left([0, T], H^{s-1} \cap H_{m l}^{r-1}(x(t), \xi(t))\right) \\
\theta \in C\left([0, T], H^{s} \cap H_{m l}^{r}(x(t), \xi(t))\right)
\end{array}\right.
$$


where the notation $u_{0} \in H_{m l}^{r}\left(x_{0}, \xi_{0}\right)$ means that there are a cutoff function $\varphi \in C_{0}^{\infty}\left(\mathbb{R}^{3}\right)$ with $\varphi\left(x_{0}\right)=1$, and a cone $K$ in $\mathbb{R}^{3} \backslash\{0\}$ about the direction $\xi_{0}$ such that $\left(1+|\xi|^{2}\right)^{r / 2} \times$ $\chi_{K}(\xi) \widehat{\varphi u_{0}}(\xi) \in L^{2}\left(\mathbb{R}^{3}\right)([1,10])$.

(2) Let $\left\{t=g_{1}(x)\right\}$ and $\left\{t=g_{2}(x)\right\}$ be the forward characteristic cones issuing from the origin for the operators $\partial_{t}^{2}-(2 \mu+\lambda) \Delta$ and $\partial_{t}^{2}-\mu \Delta$ respectively, and denote by $\mathcal{C}_{+}$the set $\left\{t>g_{1}(x)\right\} \backslash\left\{t=g_{2}(x)\right\}$. If $\left(u_{0}, \theta_{0}\right) \in H^{s}\left(\mathbb{R}^{3}\right) \cap C^{\infty}\left(\mathbb{R}^{3} \backslash\{0\}\right)$ and $u_{1} \in H^{s-1}\left(\mathbb{R}^{3}\right) \cap C^{\infty}\left(\mathbb{R}^{3} \backslash\{0\}\right)$ for $s>\frac{5}{2}$, then the local solution $(u, \theta)$ obtained above satisfies

$$
\left\{\begin{array}{l}
u \in C\left([0, T], H_{\mathrm{loc}}^{2 s-5 / 2-\epsilon}\left(\mathcal{C}_{+}\right)\right) \cap C^{1}\left([0, T], H_{\mathrm{loc}}^{2 s-7 / 2-\epsilon}\left(\mathcal{C}_{+}\right)\right) \\
\theta \in C\left([0, T], H_{\mathrm{loc}}^{2 s-5 / 2-\epsilon}\left(\mathcal{C}_{+}\right)\right)
\end{array}\right.
$$

for any $\epsilon>0$.

Sketch of The Proof. (1) As usual (see, e.g., $[11,15]$ ), by taking the decomposition

$$
u=u^{p}+u^{s}
$$

with $u^{p}$ being the potential part, $\operatorname{rot} u^{p}=0$, and $u^{s}$ the solenoidal part, $\operatorname{div} u^{s}=0$, the system (3.10) is transformed into the following one for the unknown $\left(u^{p}, u^{s}, \theta\right)$ :

$$
\left\{\begin{array}{l}
u_{t t}^{p}-\alpha^{2} \Delta u^{p}+\gamma_{1} \nabla \theta=f^{p}\left(u^{p}, u_{t}^{p}, \nabla u^{p}, u^{s}, u_{t}^{s}, \nabla u^{s}, \nabla \theta, \theta\right) \\
u_{t t}^{s}-a^{2} \Delta u^{s}=f^{s}\left(u^{p}, u_{t}^{p}, \nabla u^{p}, u^{s}, u_{t}^{s}, \nabla u^{s}, \nabla \theta, \theta\right) \\
\theta_{t}-\beta^{2} \Delta \theta+\gamma_{2} \operatorname{div} u_{t}^{p}=g\left(u^{p}, u_{t}^{p}, \nabla u^{p}, u^{s}, u_{t}^{s}, \nabla u^{s}, \theta\right)
\end{array}\right.
$$

with smooth functions $f^{p}$ and $f^{s}$, where $\alpha=\sqrt{2 \mu+\lambda}$ and $a=\sqrt{\mu}$ satisfying $\alpha>a$, and $\Delta=\sum_{j=1}^{3} \partial_{x_{j}}^{2}$. By setting

$$
\begin{cases}u_{+}^{p}=\left(\partial_{t}+i \alpha \Lambda\right) u^{p}, & u_{-}^{p}=\left(\partial_{t}-i \alpha \Lambda\right) u^{p} \\ u_{+}^{s}=\left(\partial_{t}+i a \Lambda\right) u^{s}, & u_{-}^{s}=\left(\partial_{t}-i a \Lambda\right) u^{s}\end{cases}
$$

with $\Lambda=(1-\Delta)^{1 / 2}$, and using an argument similar to Section 2 , we conclude that there is $K\left(t, x, D_{x}\right) \in \Psi^{-1}\left(\mathbb{R}^{3}\right)$ such that

$$
V=\left(V_{1}, V_{2}\right)^{T}:=\left(I+K\left(t, x, D_{x}\right)\right)\left(u_{+}^{p}, u_{-}^{p}, u_{+}^{s}, u_{-}^{s}, \theta\right)^{T},
$$

with $V_{1}$ denoting the first twelve components of $V$, satisfies the problem

$$
\left\{\begin{array}{l}
\partial_{t} V_{1}+A_{1}\left(t, x, D_{x}\right) V_{1}=B_{0}^{(1)} \cdot F_{1}\left(B_{0}^{(2)} \cdot V_{1}, B_{1} \cdot V_{2}\right) \\
\partial_{t} V_{2}-\beta^{2} \Delta V_{2}=B_{0}^{(3)} \cdot F_{2}\left(B_{0}^{(4)} \cdot V\right) \\
V_{1}(0, \cdot) \in H^{s-1}\left(R^{3}\right) \cap H_{m l}^{r-1}\left(x_{0}, \xi_{0}\right), \quad V_{2}(0, \cdot) \in H^{s}\left(R^{3}\right) \cap H_{m l}^{r}\left(x_{0}, \xi_{0}\right),
\end{array}\right.
$$

where $A_{1}\left(t, x, D_{x}\right)=\operatorname{diag}[-i \alpha \Lambda I, i \alpha \Lambda I,-i a \Lambda I$, ia $\Lambda I]$ with $I=I_{3 \times 3}$ being the $3 \times 3$ unit matrix, $B_{0}^{(k)}\left(t, x, D_{x}\right) \in \Psi^{0}\left(\mathbb{R}^{3}\right), B_{1}\left(t, x, D_{x}\right) \in \Psi^{1}\left(\mathbb{R}^{3}\right)$, and $F_{k}(\cdot)$ are smooth in their arguments. By using the classical theory of hyperbolic problems and parabolic problems, we obtain a unique solution to (3.15):

$$
\left\{\begin{array}{l}
V_{1} \in C\left([0, T], H^{s-1}\left(\mathbb{R}^{3}\right)\right) \\
V_{2} \in L^{2}\left(0, T ; H^{s+1}\left(\mathbb{R}^{3}\right)\right) \cap H^{1}\left(0, T ; H^{s-1}\left(\mathbb{R}^{3}\right)\right) .
\end{array}\right.
$$

Using (3.16) and the theory of hyperbolic equations $([1,4])$, we deduce that

$$
V_{1} \in C\left([0, T], H^{s-1}\left(\mathbb{R}^{3}\right) \cap H_{m l}^{\min (r-1, s)}(x(t), \xi(t))\right) .
$$


If $s \leq r \leq s+1$, we conclude that

$$
V_{1} \in C\left([0, T], H^{s-1}\left(\mathbb{R}^{3}\right) \cap H_{m l}^{r-1}(x(t), \xi(t))\right),
$$

which implies

$$
u \in C\left([0, T], H^{s} \cap H_{m l}^{r}(x(t), \xi(t))\right) \cap C^{1}\left([0, T], H^{s-1} \cap H_{m l}^{r-1}(x(t), \xi(t))\right) .
$$

Using (3.17) and the theory of parabolic problems gives

$$
V_{2} \in L^{2}\left(0, T ; H^{s+1} \cap H_{m l}^{r+1}(x(t), \xi(t))\right) \cap H^{1}\left(0, T ; H^{s-1} \cap H_{m l}^{r-1}(x(t), \xi(t))\right)
$$

which implies

$$
\theta \in C\left([0, T], H^{s} \cap H_{m l}^{r}(x(t), \xi(t))\right) .
$$

When $r>s+1$, we can continue this process, and conclude (3.11) eventually.

(2) For any $\left(t_{0}, x\left(t_{0}\right)\right) \in \mathcal{C}_{+}$, and $\xi\left(t_{0}\right) \in \mathbb{R}^{3} \backslash 0$, let $\tau\left(t_{0}\right) \in \mathbb{R}$ be such that $P_{0}=$ $\left(t_{0}, x\left(t_{0}\right), \tau\left(t_{0}\right), \xi\left(t_{0}\right)\right)$ is a characteristic point for the hyperbolic operators $L_{1}=\partial_{t}^{2}-$ $(2 \mu+\lambda) \Delta$ or $L_{2}=\partial_{t}^{2}-\mu \Delta$. Denote by $\gamma(t)=\left\{(t, x(t), \tau(t), \xi(t)\} \subset T^{*}\left(\mathbb{R}^{4}\right) \backslash 0\right.$ a null bicharacteristic of $L_{1}$ or $L_{2}$ passing through $P_{0}$. Obviously, the projection in $(t, x)$-space of $\gamma(t)$ intersects with $\{t=0\}$ at $x \neq 0$, where $(u, \theta)$ is smooth by using Theorem 3.1. Thus, by applying Theorem 3.2(1) we obtain

$$
\left\{\begin{array}{l}
u \in C\left([0, T], H_{m l}^{2 s-5 / 2-\epsilon}(x(t), \xi(t))\right) \cap C^{1}\left([0, T], H_{m l}^{2 s-7 / 2-\epsilon}(x(t), \xi(t))\right) \\
\theta \in C\left([0, T], H_{m l}^{2 s-5 / 2-\epsilon}(x(t), \xi(t))\right)
\end{array}\right.
$$

for any $\epsilon>0$, which is equivalent to the assertion (3.12) by using the arbitrariness of $\left(x\left(t_{0}\right), \xi\left(t_{0}\right)\right)$, because the above result holds obviously for the case that $\gamma=\{(t, x(t), \tau(t)$, $\xi(t))\}$ is a bicharacteristic of $L_{1}$ or $L_{2}$ while $\left(t_{0}, x\left(t_{0}\right), \tau\left(t_{0}\right), \xi\left(t_{0}\right)\right)$ is not a characteristic point.

3.3. Propagation of singularities in viscous fluids. In this subsection, we shall briefly explain how the decoupling idea of Section 2 can be applied to study the compressible Navier-Stokes equations with heat conduction by using paradifferential equations. It will be seen that this argument also works for general nonlinear hyperbolic-parabolic coupled equations. Denote by $\rho, u=\left(u_{1}, \ldots, u_{n}\right)^{T}, \theta, p=p(\rho, \theta)$ and $e=e(\rho, \theta)$ the fluid density, velocity, temperature, pressure and internal energy satisfying $e_{\theta}^{\prime}(\rho, \theta), k>0$ is the thermal conduction coefficient, and $\lambda, \mu$ are the Lamè constants satisfying $\lambda+\mu>0$ and $\mu>0$. The motion of the compressible viscous fluids is governed by the following Navier-Stokes equations:

$$
\left\{\begin{array}{lc}
\partial_{t} \rho+\operatorname{div}(\rho u)=0, & t>0, x \in \mathbb{R}^{n} \\
\partial_{t}\left(\rho u_{i}\right)+\operatorname{div}\left(\rho u u_{i}\right)+\partial_{i} p=\mu \Delta u_{i}+(\lambda+\mu) \partial_{i}(\operatorname{div} u), & i=1, \ldots, n \\
\partial_{t}(\rho e)+\operatorname{div}(\rho u e)+p \operatorname{div} u-k \Delta \theta=\frac{\mu}{2}\left(\partial_{i} u_{j}+\partial_{j} u_{i}\right)^{2}+\lambda(\operatorname{div} u)^{2} .
\end{array}\right.
$$

We are going to study equations (3.18) with the initial data

$$
\left.(\rho, u, \theta)\right|_{t=0}=\left(\rho_{0}(x), u_{0}(x), \theta_{0}(x)\right) .
$$

First, we recall a classical result from $[12,13]$ as follows: For any given $s>\frac{n}{2}+2$, if $\left(\rho_{0}, u_{0}, \theta_{0}\right) \in H^{s}\left(\mathbb{R}^{n}\right)$, then there is a unique local solution to (3.18), (3.19) with the 
properties:

$$
\left\{\begin{array}{l}
\rho \in C\left([0, T), H^{s}\left(\mathbb{R}^{n}\right)\right) \cap C^{1}\left([0, T), H^{s-1}\left(\mathbb{R}^{n}\right)\right) \\
u, \theta \in C\left([0, T), H^{s}\left(\mathbb{R}^{n}\right)\right) \cap C^{1}\left([0, T), H^{s-2}\left(\mathbb{R}^{n}\right)\right) \\
\nabla u, \nabla \theta \in L^{2}\left(0, T ; H^{s}\left(\mathbb{R}^{n}\right)\right) .
\end{array}\right.
$$

In order to decouple the system (3.18), we should use the paradifferential operators developed by Bony (cf. [2,5]). Given a $R \gg 1$, and $0<\epsilon_{1}<\epsilon_{2} \ll 1$, let $\chi(\theta, \eta)=$ $\phi(\theta, \eta) s(\eta)$ with

$$
s(\eta)=\left\{\begin{array}{ll}
0, & |\eta| \leq R \\
1, & |\eta| \geq 2 R,
\end{array} \quad \phi(\theta, \eta)= \begin{cases}1, & |\theta| \leq \epsilon_{1}|\eta| \\
0, & |\theta| \geq \epsilon_{2}|\eta|\end{cases}\right.
$$

The paraproduct operator $T_{a}$ is defined by

$$
T_{a} u(x)=\mathcal{F}^{-1}\left(\int \chi(\xi-\eta, \eta) \hat{a}(\xi-\eta) \hat{u}(\eta) d \eta\right)
$$

for any $a, u \in S^{\prime}\left(\mathbb{R}^{n}\right)$. With $(\rho, u, \theta)$ being given in (3.20), the equations (3.18) can be paralinearized as follows:

$$
\left\{\begin{array}{l}
\partial_{t} \rho+T_{\rho} \operatorname{div} u+\left(T_{u} \cdot \nabla\right) \rho+T_{\operatorname{div} u} \rho+\sum_{j=1}^{n} T_{\partial_{x_{j}} \rho} u_{j}=r_{\rho} \\
\partial_{t} u-T_{\rho^{-1}} L u+\left(T_{u} \cdot \nabla\right) u+T_{\rho^{-1} p_{\rho}^{\prime}} \nabla \rho+T_{\rho^{-1} p_{\theta}^{\prime}} \nabla \theta+T_{a} U=r_{u} \\
\partial_{t} \theta-T_{k /\left(\rho e_{\theta}^{\prime}\right)} \Delta \theta+\left(T_{u} \cdot \nabla\right) \theta+T_{e} \partial u+T_{b} U=r_{\theta},
\end{array}\right.
$$

where $L u=\mu \Delta u+(\lambda+\mu) \nabla(\operatorname{div} u)$ is an elliptic system, $U=(\rho, u, \theta)^{t}, a, b \in C([0, T)$, $\left.H^{s-2}\left(\mathbb{R}^{n}\right)\right)$ smoothly depend on $\left(U, \nabla U, \nabla^{2} U\right), T_{u} \cdot \nabla=\sum_{j=1}^{n} T_{u_{j}} \partial_{j}$,

$$
T_{e} \partial u:=T_{\left(p-\rho^{2} e_{\rho}^{\prime}-2 \lambda \operatorname{div} u\right) /\left(\rho e_{\theta}^{\prime}\right)} \operatorname{div} u-2 \sum_{j, k=1}^{n} T_{\mu\left(\partial_{j} u_{k}+\partial_{k} u_{j}\right) /\left(\rho e_{\theta}^{\prime}\right)} \partial_{j} u_{k}
$$

and

$$
r_{\rho} \in C\left([0, T), H^{2 s-n / 2-1}\left(\mathbb{R}^{n}\right)\right), \quad r_{u}, r_{\theta} \in C\left([0, T), H^{2 s-n / 2-2}\left(\mathbb{R}^{n}\right)\right) .
$$

In a way similar to that in Section 2 , we can find

$$
K=\left(\begin{array}{cc}
0 & -A_{1}^{(1)} A_{2}^{-1} \\
A_{2}^{-1} A_{1}^{(2)} & 0
\end{array}\right)
$$

with

$$
A_{2}=-\left(\begin{array}{cc}
T_{\rho^{-1}} L & 0 \\
0 & T_{k /\left(\rho e_{\theta}^{\prime}\right)} \Delta
\end{array}\right), \quad A_{1}^{(1)}=\left(T_{\rho} \nabla^{T}, 0\right), \quad A_{1}^{(2)}=\left(\begin{array}{c}
T_{\rho^{-1} p_{\rho}^{\prime}} \nabla \\
0
\end{array}\right),
$$

such that $V=\left(V_{1}, V_{2}\right)^{t}=(I+K) U$, with $V_{1}$ being scalar and $V_{2}$ being the last $(n+1)$ components of $V$, satisfies the weakly coupled system

$$
\left\{\begin{array}{l}
\partial_{t} V_{1}+(u \cdot \nabla) V_{1}+A_{0} V+A_{-1} U=r_{1} \\
\partial_{t} V_{2}-\left(\begin{array}{cc}
T_{\rho^{-1}} L & 0 \\
0 & T_{k /\left(\rho e_{\theta}^{\prime}\right)} \Delta
\end{array}\right) V_{2}+B_{1} V_{2}+B_{0} V+B_{-1} U=r_{2},
\end{array}\right.
$$


where

$$
\left\{\begin{array}{l}
r_{1} \in C\left([0, T), H^{2 s-n / 2-1}\left(\mathbb{R}^{n}\right)\right), \quad r_{2} \in C\left([0, T), H^{2 s-n / 2-2}\left(\mathbb{R}^{n}\right)\right) \\
B_{1} \in C\left([0, T), \operatorname{Op}\left(\Sigma_{s-n / 2-1}^{1}\right)\right)
\end{array}\right.
$$

with $\mathrm{Op}\left(\Sigma_{\sigma}^{1}\right)\left(\mathbb{R}^{n}\right)$ being the set of paradifferential operators of order one in $x$-variables (cf. $[2,5])$, and

$$
\left\{\begin{array}{l}
A_{0}, B_{0}: C\left([0, T), H^{q}\left(\mathbb{R}^{n}\right)\right) \longrightarrow C\left([0, T), H^{q}\left(\mathbb{R}^{n}\right)\right) \\
A_{-1}, B_{-1}: C\left([0, T), H^{q}\left(\mathbb{R}^{n}\right)\right) \longrightarrow C\left([0, T), H^{q+1}\left(\mathbb{R}^{n}\right)\right)
\end{array}\right.
$$

are bounded for any $q \in \mathbb{R}$.

REMARK 3.2. To see what $V_{2}$, the part of $U=(\rho, u, \theta)^{T}$ having smoothing effect, is, let us consider the equations of isothermal fluids, i.e. we do not need the equation for the balance of energy in (3.18). Then, by direct computation, we deduce that

$$
\mu \Delta V_{2}+(\lambda+\mu) \nabla\left(\operatorname{div} V_{2}\right)=\nabla F-\mu \operatorname{rot}(\operatorname{rot} u)+\cdots \ldots ",
$$

where the dots represent some terms smoother than the first two terms on the right-hand side, and

$$
F=(\lambda+2 \mu) \operatorname{div} u-p(\rho)
$$

is the effective viscous flux, which was throughly studied by D. Hoff et al. $([8,9])$.

Similarly to Theorem 3.2(2), by using the theory of hyperbolic equations and a classical bootstrap argument for the equations (3.22), we can establish the following result:

Theorem 3.3. For any fixed $\frac{n}{2}+2<s \leq r \leq 2 s-\frac{n}{2}$, suppose that

$$
\left(\rho_{0}, u_{0}, \theta_{0}\right) \in H^{s}\left(\mathbb{R}^{n}\right) \cap H_{m l}^{r}\left(x_{0}, \xi_{0}\right)
$$

with a fixed $\left(x_{0}, \xi_{0}\right) \in T^{*}\left(\mathbb{R}^{n}\right) \backslash 0$. Let $u \in C\left([0, T), H^{s}\left(\mathbb{R}^{n}\right)\right) \cap C^{1}\left([0, T), H^{s-2}\left(\mathbb{R}^{n}\right)\right)$ be the velocity component of the unique local solution of $(3.18)$, and $\gamma(t)=(t, x(t) ; \tau(t), \xi(t))$ $(0 \leq t<T)$ be a null bicharacteristic of $\partial_{t}+u \cdot \nabla$ with $x(0)=x_{0}$ and $\xi(0)=\xi_{0}$. Then the unique solution $(\rho, u, \theta)$ of (3.18) satisfies

$$
(\rho, u, \theta) \in C\left([0, T), H^{s} \cap H_{m l}^{r}(x(t), \xi(t))\right) .
$$

REMARK 3.3.

(1) The detail of the proof for Theorem 3.2 can be found in $[6,19]$, and the detail of Theorem 3.3 can be found in [7]. Moreover, in [7], we have also obtained a result on the propagation of microlocal Hölder regularity for the Navier-Stokes equations (3.18).

(2) Recently, in [9], D. Hoff obtained an interesting result on the propagation of discontinuities in compressible Navier-Stokes equations of isothermal fluids in two space dimensions.

(3) Some other interesting applications of the decoupling idea can be developed as well. In [20], we have partially applied this idea to study the $L^{p}-L^{q}$ decay rate of solutions to Cauchy problems for linear thermoelastic systems with time-dependent coefficients. In [14], we use this decoupling idea to study the global existence of smooth solutions to the Navier-Stokes equations. 
Acknowledgements. The results on propagation of singularities in thermoelasticity and in compressible viscous fluids were collaborated with Chen Shuxing. The author would like to express his gratitude to Michael Reissig for fruitful discussions.

\section{References}

[1] M. Beals, Propagation and Interaction of Singularities in Nonlinear Hyperbolic Problems, Birkhäuser, Boston 1989.

[2] J. M. Bony, Calcul symbolique et propagation des singularités pour les équations aux dérivées partielles nonlinéaires, Ann. Sci. École Norm. Sup. (4) 14 (1981), 209-246.

[3] J. Chazarain and A. Piriou, Introduction to the Theory of Linear Partial Differential Equations, North-Holland, Amsterdam, 1982.

[4] S. X. Chen, Analysis of Singularities for Partial Differential Equations (in Chinese), Shanghai Scientific \& Technical Publishers, 1998.

[5] S. X. Chen, Q. QIu and C. Li, Introduction to Paradifferential Operators (in Chinese), Sci. Publishers, Beijing, 1990.

[6] S. X. Chen and Y. G. WAng, Propagation of singularities of solutions to hyperbolic-parabolic coupled systems, Math. Nachr. 242 (2002), 46-60.

[7] S. X. Chen and Y. G. WAng, Propagation of singularities in compressible viscous fluids, in: Geometry and Nonlinear Partial Differential Equations (Hangzhou, 2001), AMS/IP Stud. Adv. Math. 29, Amer. Math. Soc., Providence, 2002, 13-25.

[8] D. HofF, Global solutions of the Navier-Stokes equations for multidimensional compressible flow with discontinuous initial data, J. Differential Equations 120 (1995), 215-254.

[9] D. HofF, Dynamics of singularity surfaces for compressible, viscous flows in two space dimensions, Comm. Pure Appl. Math. 55 (2002), 1365-1407.

[10] L. Hörmander, The Analysis of Linear Partial Differential Operators I, Springer, Berlin, 1983.

[11] S. JIANG and R. RACKE, Evolution Equations in Thermoelasticity, Chapman Hall/CRC Monogr. Surv. Pure Appl. Math. 112, Chapman \& Hall, Boca Raton, 2000.

[12] P.-L. Lions, Mathematical Topics in Fluid Mechanics, vol. 2, Compressible Models, The Clarendon Press, Oxford Univ. Press, New York, 1998.

[13] A. Matsumura and T. Nishida, Initial boundary value problems for the equations of motion of compressible viscous fluids, in: Nonlinear Partial Differential Equations (Durham, 1982), J. A. Smoller (ed.), Contemp. Math. 17, Amer. Math. Soc., Providence, 1983, 109-116.

[14] M. Padula and Y. G. WANG, A new approach on the existence of nonsteady motions for a viscous compressible fluid, in preparation.

[15] R. RACKE, Lectures on Nonlinear Evolution Equations. Initial Value Problems, Aspects Math. E19, Vieweg, Braunschweig, 1992.

[16] R. RACKe and Y. G. WANG, Propagation of singularities in one-dimensional thermoelasticity, J. Math. Anal. Appl. 223 (1998), 216-247.

[17] M. REIssig and Y. G. WANG, Propagation of mild singularities in higher-dimensional thermoelasticity, J. Math. Anal. Appl. 240 (1999), 398-415.

[18] M. TAYLOR, Reflection of singularities of solutions to systems of differential equations, Comm. Pure Appl. Math. 28 (1975), 457-478.

[19] Y. G. WANG, Microlocal analysis in nonlinear thermoelasticity, Nonlinear Anal. (to appear).

[20] Y. G. WANG and M. ReISsig, Influence of the hyperbolic part on decay rates in 1-d-thermoelasticity, in: Proceedings in honour of Prof. J. Vaillant, Kluwer (to appear).

[21] S. Zheng, Nonlinear Parabolic Equations and Hyperbolic-Parabolic Coupled Systems, Pitman Monogr. Surveys Pure Appl. Math. 76, Longman, Harlow, 1995. 